\title{
Trattamento di paziente emorragico in reparto di terapia intensiva (ICU)
}

\author{
D. Zazzaro, R. Colombo', S. Pennacchia ${ }^{2}$, L. Delfino ${ }^{2}$, G. Splendiani \\ Cattedra Nefrologia Università Tor Vergata Roma, \\ ${ }^{1}$ Servizio Emodialisi Aurelia Hospital Roma, \\ ${ }^{2}$ Servizio Rianimazione Aurelia Hospital Roma
}

\section{P}

aziente di anni 48 , maschio, con uremia terminale in trattamento emodialitico ambulatoriale dal 1982, presenta un episodio di embolia polmonare: va in arresto cardiorespiratorio per fibrillazione ventricolare. Viene pertanto sottoposto a cardioversione elettrica a $200 \mathrm{~J}$., poi a cardioversione farmacologica con boli di lidocaina, fino al ripristino del ritmo sinusale. Essendo in arresto respiratorio viene intubato e ventilato con Ambu, quindi trasferito in ICU (Intensive Care Unit) dopo esecuzione di TC cranio. Viene collegato a ventilatore meccanico in SIMV (Sincronized Intermittent Mandatory Ventilation) e monitorizzato. All'esame obiettivo neurologico presenta segni di decerebrazione, a livello circolatorio PAM (pressione arteriosa media) di $60 \mathrm{mmHg}$ : pertanto viene assistito farmacologicamente con dopamina e dobutamina in infusione continua, oltre ad infusione di sostanze iperosmotiche, steroidee ed anti- $\mathrm{H}_{2}$. Si decide per CRRT (Continuous Renal Replacement Therapy) in CVVHD (Continuous Veno-Venous Hemodialysis).

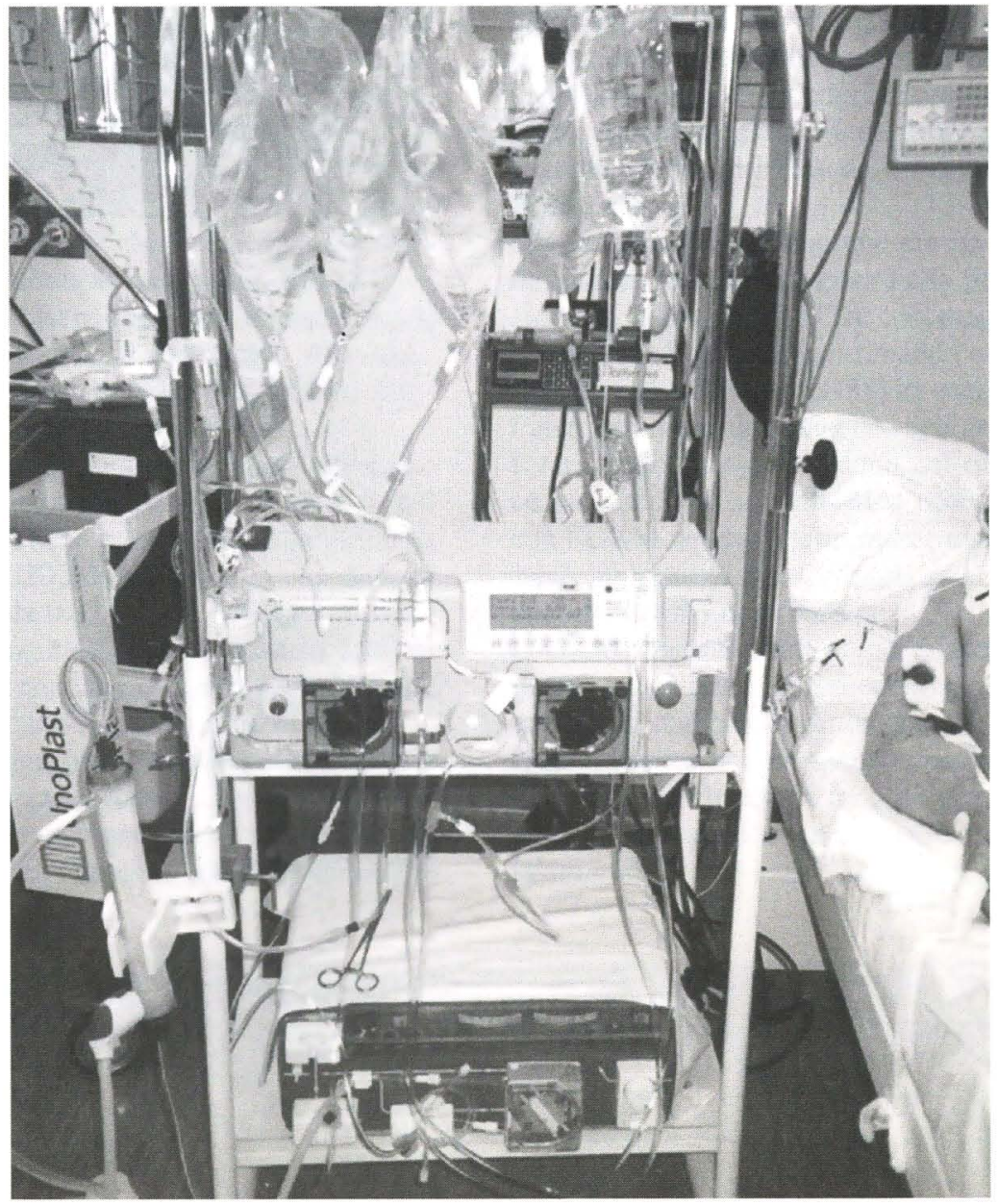

Fig. 1 


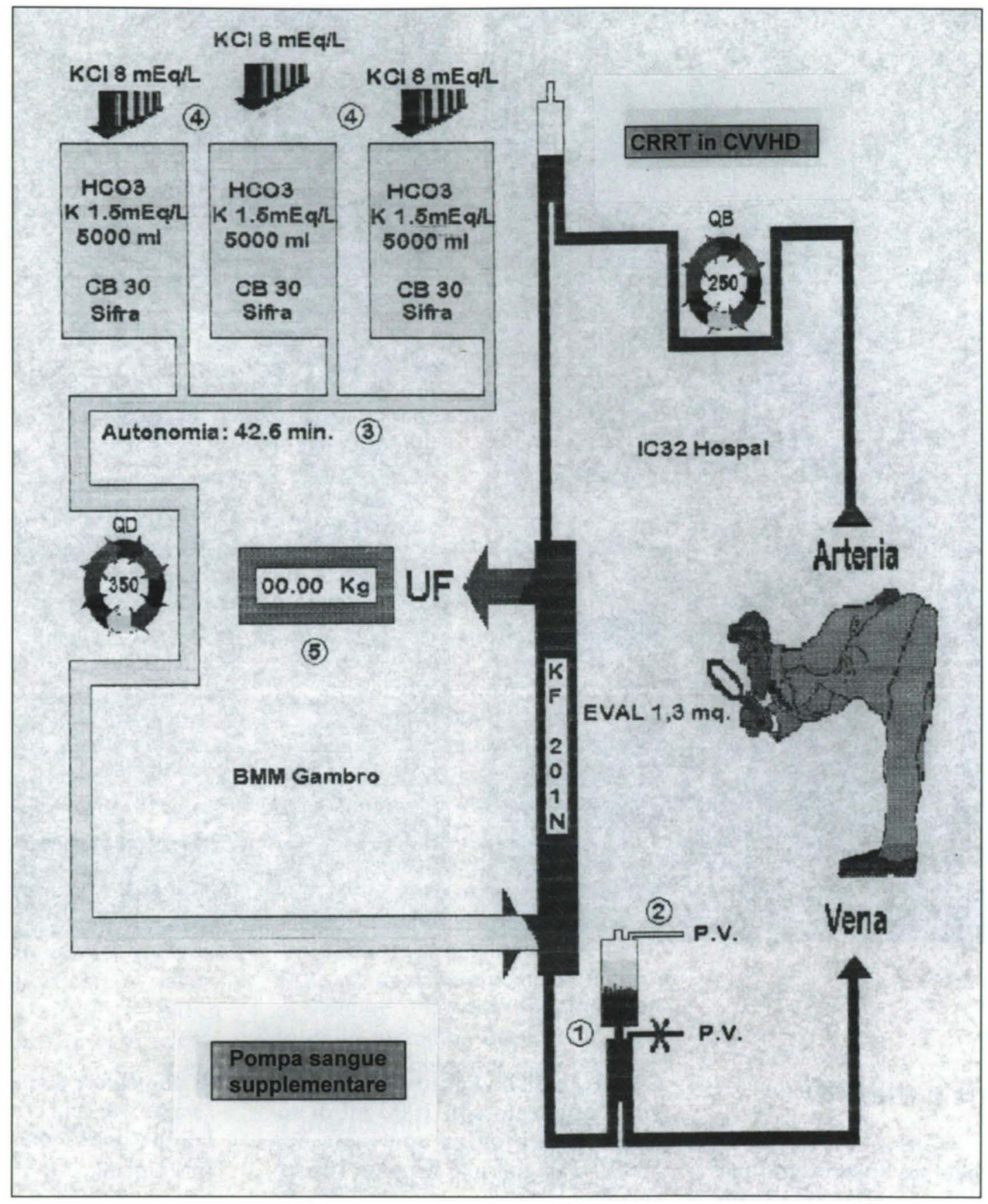

Fig. 2

Il trattamento viene continuato per 21 giorni (504h) fino a quando il paziente manifesta melena ed ematemesi. Una gastroscopia eseguita d'urgenza rivela la presenza di una ulcera duodenale sanguinante. Al paziente vengono quindi somministrati antiemorragici e sospesa la terapia steroidea; inoltre viene iniziato ciclo trasfusionale. Si continua il trattamento CVVHD in regionale, con infusione di solfato di protamina per 6 giorni (144h), senza però miglioramento dello stato emorragico. Una ulteriore gastroscopia di controllo conferma la presenza di uno zampillo di sangue arterioso a livello duodenale. Viene quindi effettuato un trattamento a bassa efficienza in EVAL. In previsione di un intervento chirurgico risolutivo si rende però necessario affrontare il problema di un trattamento dialitico ad alta efficienza, senza eparina. La grave emorragia in atto esclude la possibilità di inserire tecniche emodiafiltrative in continua; si ricorre quindi ad una tecnica alternativa consistente nella aggiunta di una pompa di reinfusione al modello IC 32 Hospal da CRRT, in modalità EVAL (Etilen-Vinil-Alcool) eseguita in ICU (Fig. 1). 


\section{$x$

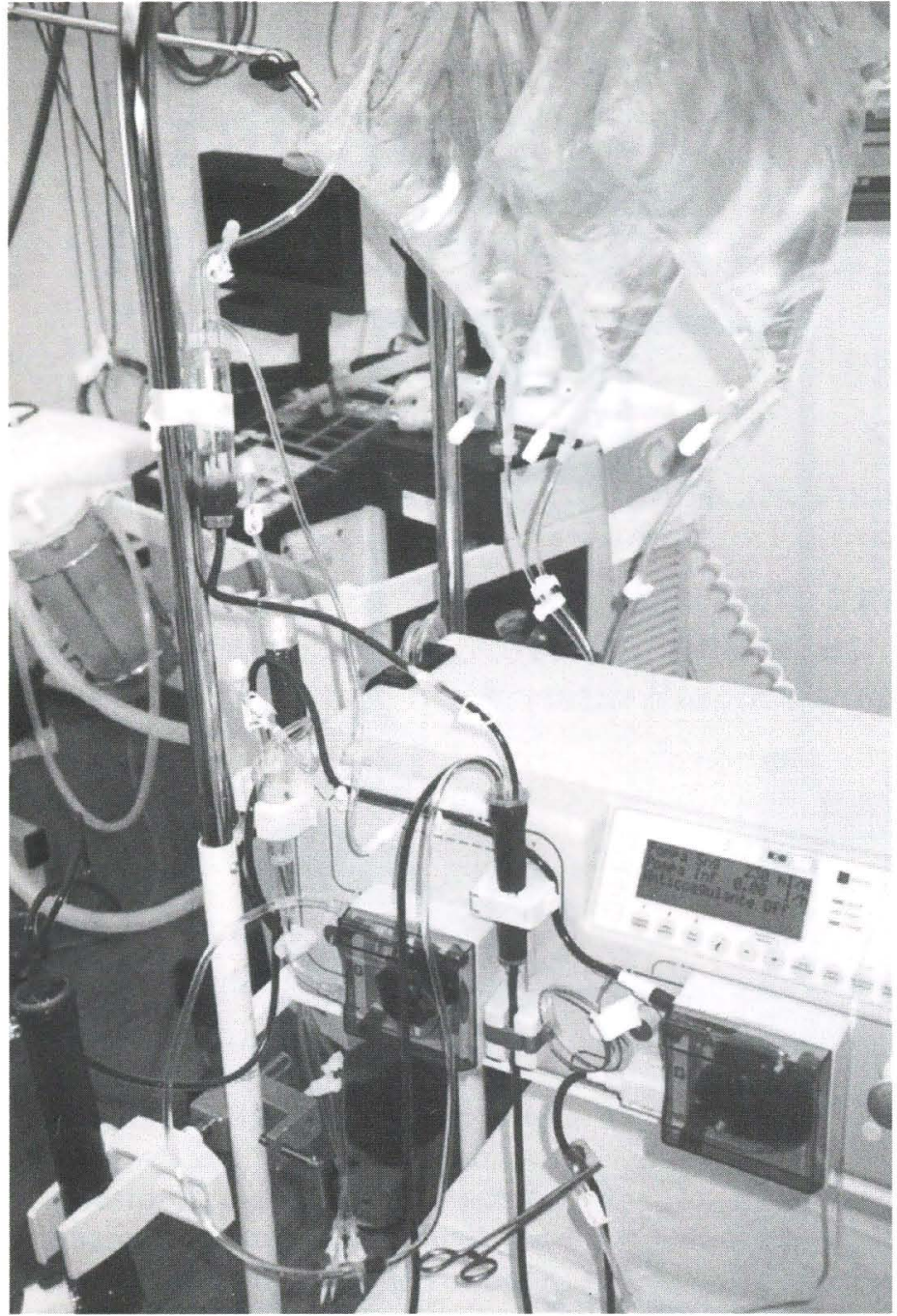

Fig. 3

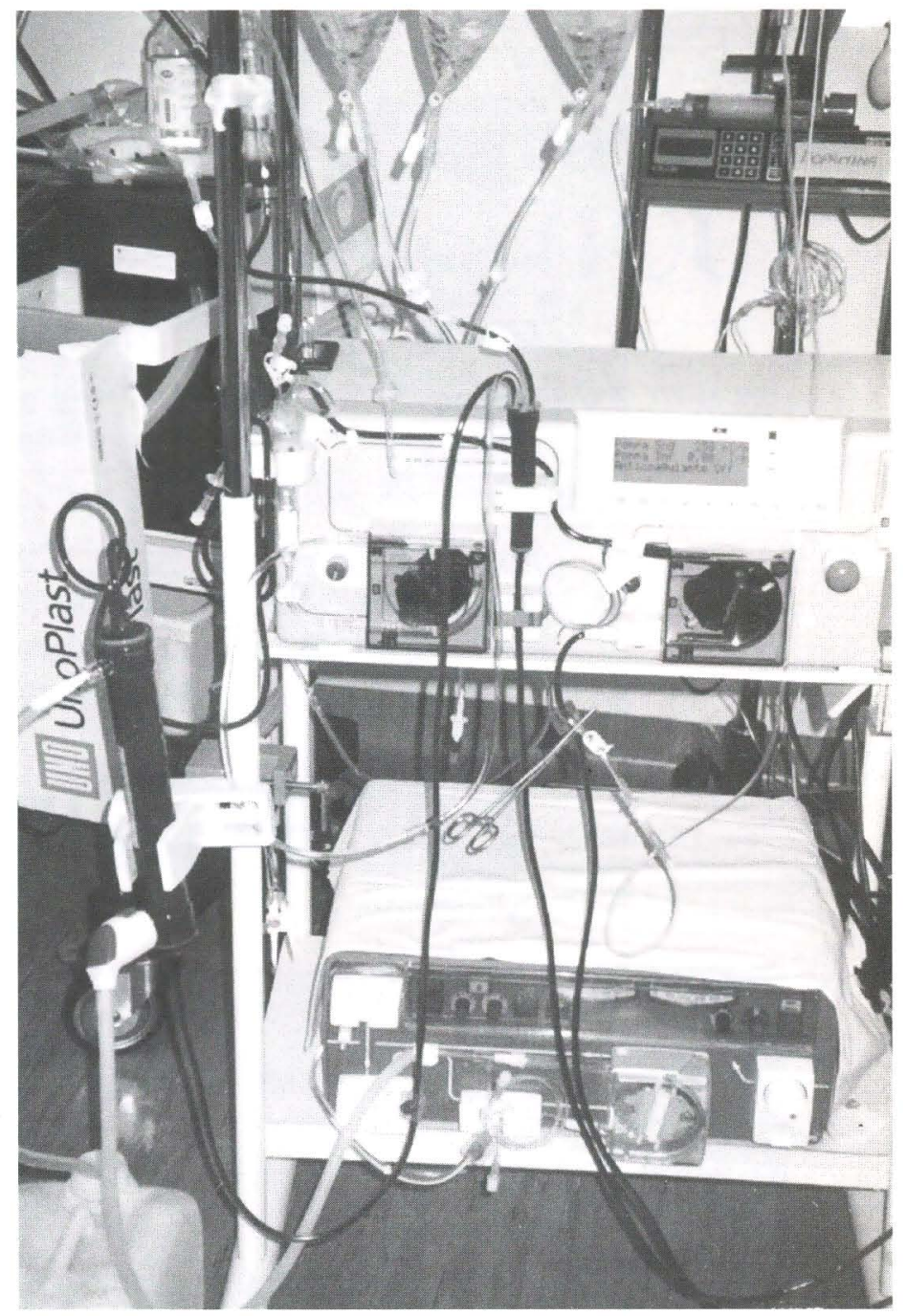

Fig. 4

\section{Materiali e metodi}

Viene impiegato un apparecchio per CRRT modello Hospal IC32, un modulo BMM Gambro, un filtro Bieffe KF-201N (EVAL) con superficie $1.3 \mathrm{~m}_{2}$, sacche di soluzione bicarbonato Sifra tipo CB 30 da 5000 $\mathrm{ml}$ a $\mathrm{K} 1.5 \mathrm{mEq} / \mathrm{L}$ e $\mathrm{HCO}_{3} 30$ $\mathrm{mEq} / \mathrm{L}$. Si prepara dapprima il Circuito dell'IC32, raccordando il filtro EVAL alle linee e connettendo un secondo pozzetto piezometrico (punto 1 Fig. 2) al pozzetto venoso: ciò al fine di evitare il contatto diretto del sangue del paziente con l'aria. L'IC32 viene impostata in modalità
CVVHD. Il lavaggio del circuito viene effettuato con $1000 \mathrm{ml}$ di soluzione fisiologica eparinata con 5000 UI di eparina, rimossa successivamente dal lavaggio del circuito con altri $2000 \mathrm{ml}$ di soluzione fisiologica. La lettura della pressione venosa viene effettuata dal sensore del secondo pozzetto piezometrico (punto 2 Fig. 2), l'unico in contatto con l'aria. Si prepara quindi il Circuito accessorio (BMM Gambro), necessario per aspirare soluzione bicarbonato alla velocità di $350 \mathrm{ml} / \mathrm{m}$ (QD) da infondere al filtro. Essendo l'autonomia di una sacca, a tale QD, di soli 14.2 minuti, si è posta la necessità, lavorando in un reparto ICU, di aumentare i tempi di durata delle sacche stesse, creando un artificio tale da poter connettere, contemporaneamente, tre sacche in serie con una autonomia di 42.6 minuti (punto 3 Fig. 2). Essendo il paziente in una condizione di ipokaliemia, vengono aggiunte alle sacche bicarbonato 8 $\mathrm{mEq} / \mathrm{L}$ di $\mathrm{KCl}$, al fine di ottenere una concentrazione finale di 3.1 $\mathrm{mEq} / \mathrm{L}$ (punto 4 Fig. 2). La linea del circuito dialisato viene connessa al filtro EVAL in controcorrente al sangue e l'ultrafiltrato viene raccolto in apposita tanica posizionata su bilancia digitale per la valutazione corretta delle entrate/uscite (punto 5 Figs. 2, 3, 4). 


\section{Risultati}

\begin{tabular}{lcc} 
EVAL modificato & Iniziale & Finale \\
\hline Attività protrombinica & $97 \%$ & $97 \%$ \\
aPTT & $32 "$ & $32 "$ \\
INR & 1.02 & 1.03 \\
Fibrinogeno & 513 & 516 \\
Azotemia & 251 & 75 \\
B.E. & -3.2 & +1.5 \\
$\mathrm{HCO}_{3}$ & 18 & 24 \\
$\mathrm{~K}$ & 3.1 & 3.3 \\
$\mathrm{Na}$ & 125 & 134 \\
\hline
\end{tabular}

\section{Discussione}

L'impossibilità ad impiegare sostanze eparinate per il mantenimento del circuito extracorporeo in CRRT, in un paziente uremico cronico con problemi emorragici in atto, collegato a ventilatore meccanico e con la necessità di una depurazione ottimale per poter essere sottoposto ad intervento chirurgico, poneva sicuramente indicazione, con carattere d'urgenza, a trattamento emodialitico ad alta efficienza, senza eparina. Nella impossibilità del trasferimento del paziente in una unità di emodialisi tradizionale, con impianto d'osmosi inversa (vista la situazione logistica preesistente), si imponeva la necessità di una scelta metodologica che soddisfacesse tutte le esigenze. Pertanto si poneva sicuramente indicazione a trattamento emodialitico con EVAL, da effettuare in ICU, cercando però di migliorare ed aumentare la capacità diffusiva e quindi depurativa del sistema. A tal fine si sottoponeva il paziente a trattamento emodialitico per 210 minuti, impiegando un flusso sangue (QB) di $250 \mathrm{ml} / \mathrm{m}$.; si impiegava quindi un circuito accessorio (pompa Gambro) per mandare al filtro un QD di $350 \mathrm{ml} / \mathrm{m}$. di soluzione tampone bicarbonato, eliminando quindi il problema legato alla mancanza di impianto d'osmosi inversa per il trattamento dell'ac- qua di rete. Lo scambio in tal modo era di 21 litri/h, contro i 30 litri/h di un trattamento di emodialisi tradizionale. L'impiego del filtro EVAL permetteva di non utilizzare sostanze eparinate, mentre la somministrazione quale dialisato di sostanza tampone bicarbonato, permetteva il mantenimento di una stabilità cardiovascolare in un paziente critico (PAM $60 \mathrm{mmHg}$ ), oltre che una buona correzione dello stato di acidosi metabolica uremica. Il monitoraggio dell'EGA, dell'esame emocromocitometrico, degli elettroliti della azotemia pre/post, permetteva di valutare il corretto funzionamento della metodica impiegata. Il paziente effettuava nel pre-operatorio 1 trattamento e nel post-operatorio 2 trattamenti in EVAL modificato, nell'arco di tempo di 6 giorni: il filtro a fine dialisi rimaneva pulito. A risoluzione del problema acuto emorragico, veniva reinserito in CRRT.

\section{Conclusioni}

La metodica impiegata, pur nella sua semplicità, appare risolutiva in situazioni critiche emorragiche di pazienti uremici, ricoverati in ICU, che richiedano alta efficienza depurativa. I costi di gestione di tale metodica non incidono sui costi com- plessivi del paziente critico più di quelli dello stesso in CRRT: una giornata di CRRT infatti ha un costo di circa L. 470.000/die, contro le L. 344.000 della metodica in EVAL modificata, con carattere per di più intermittente.

Pertanto a nostro avviso può rappresentare un utile ausilio alla CRRT in situazioni emorragiche di pazienti uremici in ICU. 